\title{
Delta Wing's Detached Vortex Fracture Control and PIV Analysis
}

\author{
Rui Zhu \\ Department of Aeronautics, Xiamen University, Xiamen 361005, China \\ zhurui@xmu.edu.cn
}

Keywords: Vortex generator, delta wing, flow visualization, Particle Image Velocimetry detached vortex.

\begin{abstract}
This paper study's the influence of vortex generator on the breakpoint of delta wings detached vortex. Experimental research on the interaction between vortex generator created auxiliary vortex and delta wing's detached vortex is conducted under different flow speed and attack angles. Flow visualization test is to study the physical mechanisms of development and fracture of detached vortex. The Time-resolved PIV (Particle Image Velocimetry) processing is undertaken to achieve the characteristics of interaction, development and fracture of the delta wing's detached vortex and generator created vortex. The advantageous impact of vortex generator on delta wing's detached vortex is revealed. The analysis of PIV processed velocity and vortices fields verifies an explains the flow visualization experiments results.
\end{abstract}

\section{Introduction}

The delta wing design is widely used in modern jet fighter. The detached vortices from delta wing can increase lift and improve aircraft comprehensive performance, and solve the contradiction of wing shape requirements of subsonic, transonic and supersonic [1]. Modern fighter use various type of delta wing or transformed delta wing design that fully utilize big attack angle detached vortices to generate non-linear lift, namely effectively use the ample vortices flow structure in the flow field [2]. Based on the complete understanding of the characteristics of delta wing flow field, study and control vortices separations and interaction, create an optimal delta wing structure design with vortex generator will place a significant value to national military aviation industry.

\section{Delta Wing Lift Characteristics}

Delta wing can delay the boundary layer separation, this characteristic indicate that a pair of counter-rotating concentrated vortices generated from delta wing leading edge (with a certain angle) [3]. The concentrated vortex induces high-speed air flow in local area above surface, thus create low pressure area above wing surface and under vortex core, and form a non-linear suction peak area on surface [4]. The distribution of wing surface suction peak is closely related to vortices' space positions, shown in Fig.1. The suction peak area generates the non-linear vortex lift, and this extra "vortex lift" increases the wing lift [5].

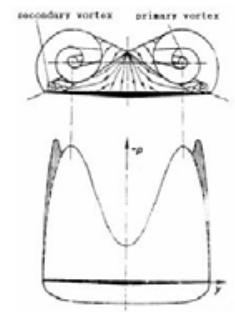

Fig. 1 Separation vortices and surface pressure

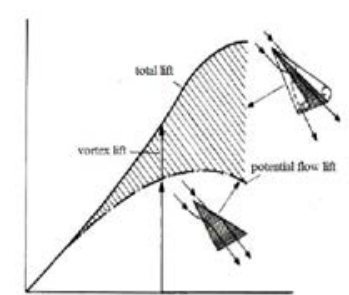

Fig. 2 Delta wing lift coefficient curve 


\section{Experiments Requirements}

Experimental Facilities. The experiments are undertaken in the Fluid Mechanics and PIV Laboratory of Xiamen University. The FMPL now has multi-functional precision recycling water channel system (Fig.3) and PIV system. The water channel system can provide stable and recyclable water flow field for PIV system. The PIV apparatus consists of a camera, a laser with an optical arrangement to limit the physical region illuminated, a synchronizer to act as an external trigger for control of the camera and laser, the seeding particles and the fluid under investigation. PIV software is used to post-processes the optical images.

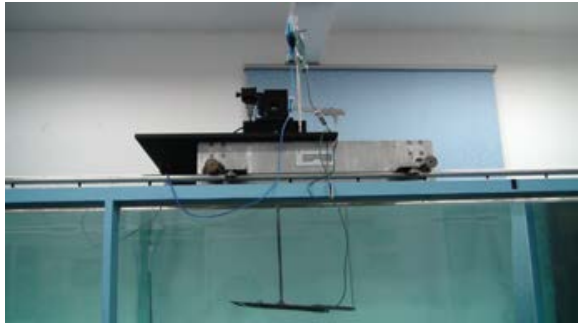

Fig. 3 Water channel system

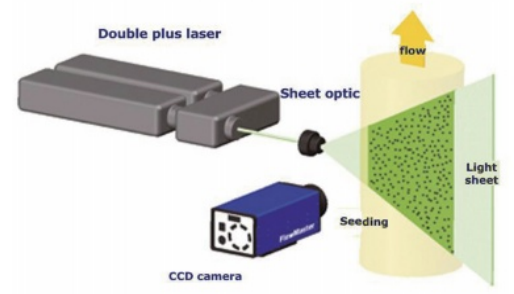

Fig. 4 Particle image velocimetry

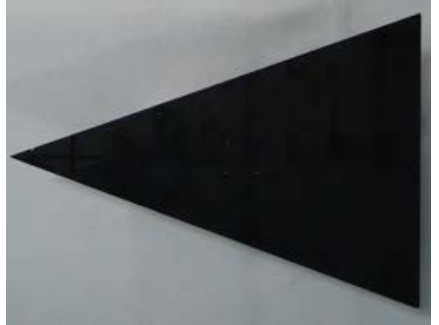

Fig. 5 Experimental model

Experimental Model. The experimental model used is shown in Fig.5. The experimental model parameters: backswept angle $70^{\circ}$, root length $300 \mathrm{~mm}$. The delta wing's detached vortex experiments are conducted with and without vortex generator to study its impact on primary vortex fracture point.

\section{Attack Angles' Influence on Detached Vortex Fracture}

The visualization experiments are done under different attack angles $\left(10^{\circ}, 15^{\circ}, 20^{\circ}, 25^{\circ}, 30^{\circ}, 35^{\circ}\right)$, and flow speed $0.2 \mathrm{~m} / \mathrm{s}$. The experiments results shown in Fig. 6 indicate the detached vortex fracture point. From Fig.6, when flow speed is $0.2 \mathrm{~m} / \mathrm{s}$, the detached vortex fracture point position moves toward to front wingtip as attack angle increases. When attack angle $\alpha=10^{\circ} \& 15^{\circ}$, the fracture point is about at aft $7 \mathrm{~cm}$ to delta wing trailing edge; When $\alpha=25^{\circ}$, fracture point is at 0.8 root length; When $\alpha=35^{\circ}$, fracture point is at 0.2 root length. Obviously, the fracture point moves to front wingtip as attack angle increases.

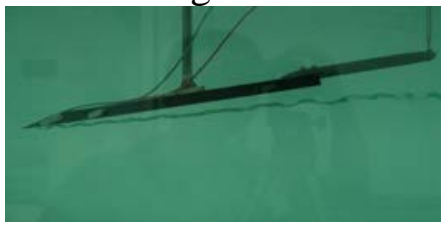

$\alpha=10^{\circ}$

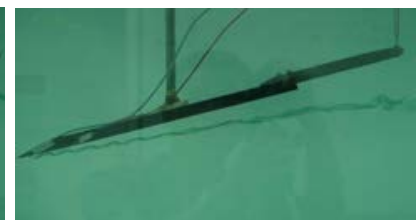

$\alpha=15^{\circ}$

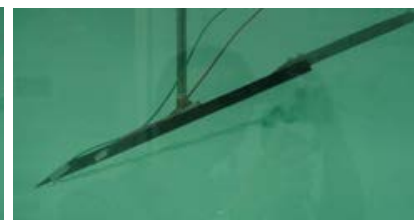

$\alpha=25^{\circ}$

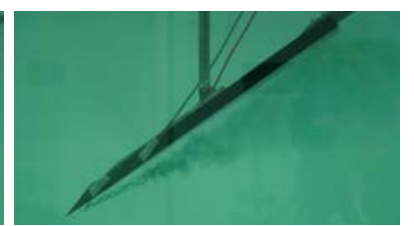

$\alpha=35^{\circ}$

Fig. 6 Fracture points under different attack angles

\section{Vortex Generator's Influence on Detached Vortex Fracture}

The delta wing's detached vortex easily disturbed by exterior factors, two vortex generator installed on delta wing to generate counter-rotating and co-rotating vortices. The counter-rotating vortex will weaken primary vortex. The co-rotating vortex will enhance primary vortex to delay primary vortex fracture.
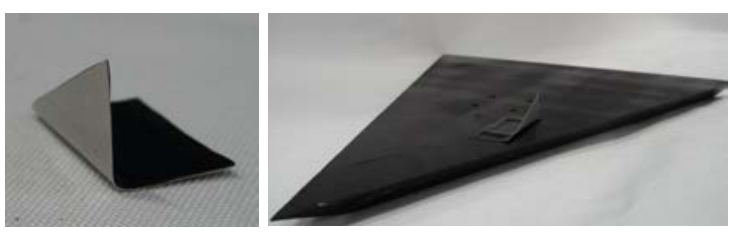

Fig. 7 Counter-rotating vortex generator

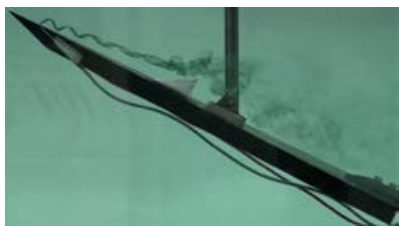

with generator

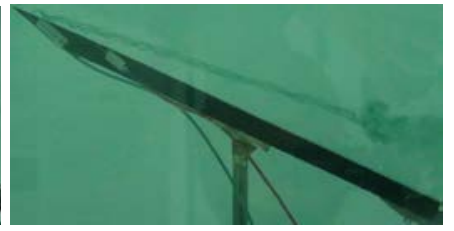

no generator

Fig. 8 Fracture point positions

Counter-rotating Vortex's Influence on Detached Vortex Fracture. The counter-rotating vortex generator and installation are shown in Fig.7. The generated counter-rotating vortex tangles with the primary vortex, the two vortices' rotation is contrary but the primary vortex strength is much 
higher, the generated counter-rotating vortex "swallowed" by primary vortex that causes fracture point to move ahead due to primary vortex strength reduction. Fig.8 shows the comparison between fracture point positions of with and without vortex generator.

Co-rotating Vortex's Influence on Detached Vortex Fracture. Experiments with different experimental parameters (vortex generator size, angle, position) reveal that there are two main influence caused by co-rotating vortex generator: the primary vortex fracture point position extended because the generated vortex strength higher than generator's hindering effect thus enhance primary vortex energy; the fracture point position moves ahead for the generator's hindering effect is stronger and weakens the primary vortex. The co-rotating vortex generator and installation are determined (shown in Fig.10) after many experiment attempts to assure enhanced primary vortex and delay the primary vortex fracture. Fig.9 indicates the delta wing primary vortex fracture point position extended with generator influence under different $\alpha, v$.

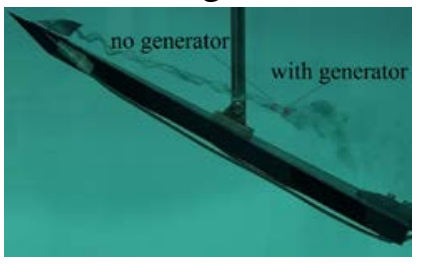

(1) $\alpha=25^{\circ}, v=0.2 \mathrm{~m} / \mathrm{s}$

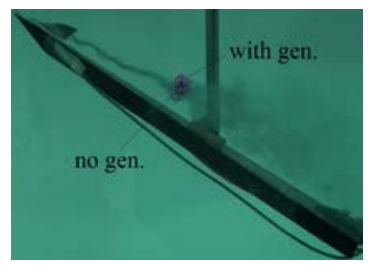

(2) $\alpha=30^{\circ}, v=0.2 \mathrm{~m} / \mathrm{s}$

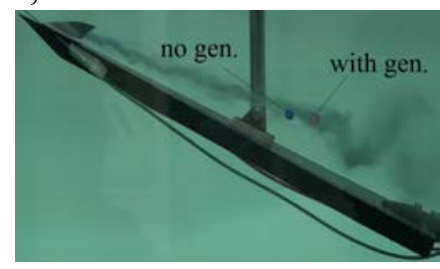

(3) $\alpha=25^{\circ}, v=0.3 \mathrm{~m} / \mathrm{s}$

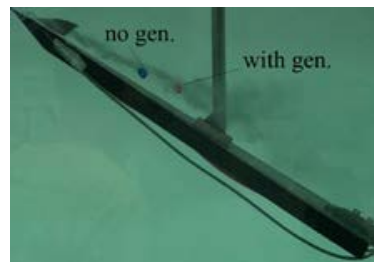

(4) $\alpha=30^{\circ}, v=0.3 \mathrm{~m} / \mathrm{s}$

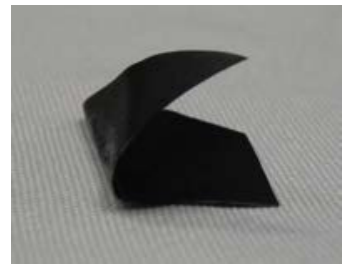

Fig. 10 Co-rotating vortex generator

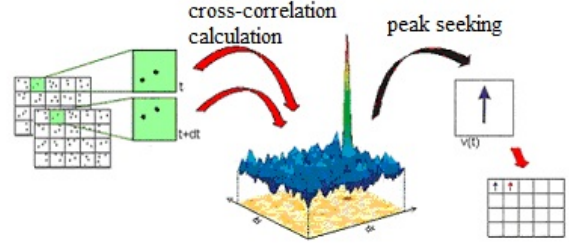

Fig. 11 PIV Cross-correlation Analysis

In experiment (1), (3), (4), the co-rotating vortex generator has less impact on the primary vortex stall, and creates stable enhance to primary vortex energy, the fracture point obviously extended. Experiments reveal that the co-rotating vortex generator can always delay the fracture under proper installation with a particular attack angle and a flow speed.

\section{Detached Vortex PIV Analysis}

PIV Cross-correlation Analysis. In PIV cross-correlation analysis, the frames are split into a large number of interrogation areas, or windows. It is then possible to calculate a displacement vector for each window with help of signal processing and cross-correlation techniques. This is converted to a velocity using the time between laser shots and the physical size of each pixel on the camera. The size of the interrogation window should be chosen to have at least 6 particles per window on average (Fig.11).

PIV Processing of Detached Vortex Flow Field Section. The PIV processing is conducted under conditions of with and without vortex generator, and the secondary vortices developments and interaction with the primary vortex under these conditions are compared and analyzed. Fig.12 (a) shows two secondary vortices at "1" and "2" position in no generator condition. During dynamic

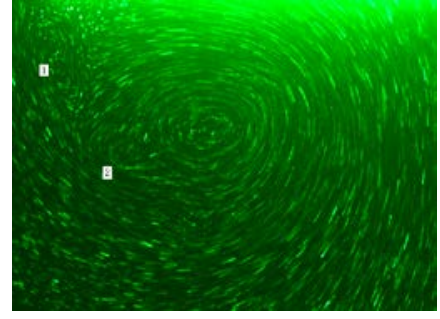

(a) no generator left side

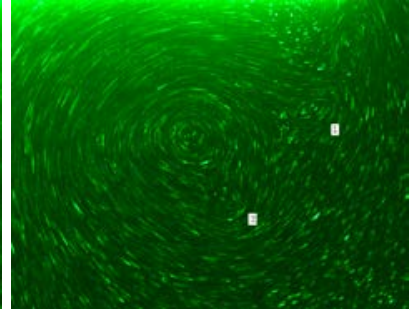

(b) with generator right side

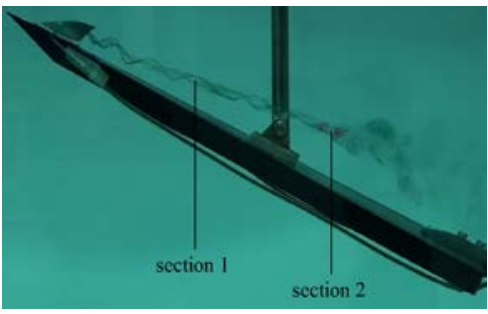

Fig. 12 PIV processing of flow field section Fig. 13 Section 1 and 2 for PIV processing 
Experiment process, the secondary vortices co-rotate with the primary vortex, move toward the primary vortex and eventually "swallowed" by primary vortex thus enhance the primary vortex. Fig.12 (b) shows the condition with vortex generator. The secondary vortices at "1" and "2" position are stable and almost static because of the high frequency of secondary vortices generation and merger with the primary vortex.

PIV Analysis of Vortex Fracture. Under $\alpha=25^{\circ}, \mathrm{v}=0.2 \mathrm{~m} / \mathrm{s}$, PIV processing done for section 1 and section 2 shown in Fig.13. The PIV processed velocity field of section 1 and section 2 (Fig.14) indicates that: left side (without generator) and right side (with generator) velocity field are both closed at section 1 because both sides' vortices haven't fracture; The left side velocity field turns unclosed and the right side's still closed at section 2 because the left side vortex already fracture but right side's hasn't.

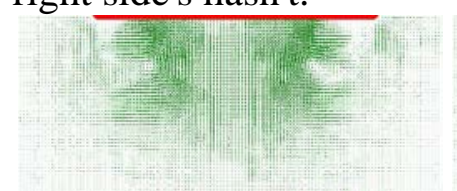

Section 1

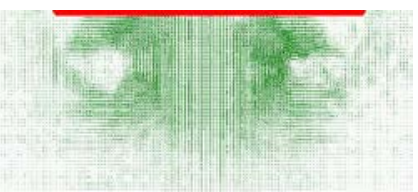

section 2

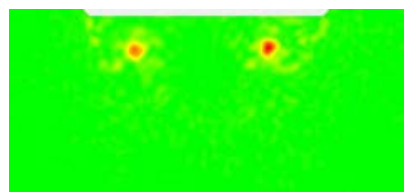

section 1

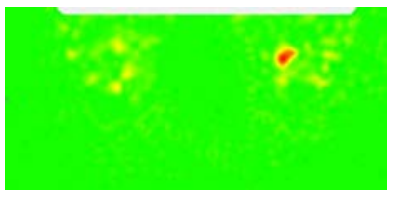

section 2

Fig. 14 Velocity field of section 1 and 2

Fig. 15 Energy reprogram of section 1 and 2

The curl of velocity field is vorticity, is two times of the angular velocity of any micelle in fluid field, vorticity represents the vortex energy. Fig. 15 shows the energy reprogram of section 1 and 2. Obviously, at section 1 both sides' vorticity fields maintain relatively good energy because both sides' vortices haven't fracture; At section 2, left side vorticity field already dissipate but the right side still maintains good energy for the left side vortex already fracture but right side's hasn't. The generated vortex enhances the primary vortex energy and makes the primary vorticity field last longer. The same experiments results also come from other two parameters sets: $\alpha=25^{\circ}, \mathrm{v}=0.3 \mathrm{~m} / \mathrm{s} ; \alpha=30^{\circ}$, $\mathrm{v}=0.3 \mathrm{~m} / \mathrm{s}$.

\section{Conclusions}

1. There is a particular area on delta wing where vortex generator installed can cause the primary vortex fracture delay.

2. The reasons for primary vortex fracture to occur earlier: vortex generator created vortex counter-rotates with primary vortex; the vortex generator has hindering effect on primary vortex generation and development.

3. The reasons why the primary vortex fracture delay happens are: the generated vortex co-rotates with primary vortex; generated vortex's strengthening effect to primary vortex is stronger than generator's hindering effect.

4. PIV processing verifies and explains the flow visualization experiments results based on velocity fields and vorticity fields analysis.

\section{References}

[1] Babie Brian M., Nelson Robert C.: The identification of an unstable wake vortex. J. Journal of Flow Visualiza-tion and Image Processing, v 16, n 2, p 103-125 (2009).

[2] Leweke Thomas, Williamson Charles H. K.: Experiments on long-wavelength instability and reconnection of a vortex pair. J. Physics of Fluids, v 23, n 2, February 18 (2011).

[3] Bao. F, Vollmers.H. Alleviation of End-effect in Facili-ties for Far Wake Investigations. In: 43rd AIAA Aero-space Sciences Meeting and Exhibit, Reno, Nevada, USA (2005).

[4] Heyes A.L., Taylor N.P., and Chen J.: Characterising short-wave instabilities on a vortex dipole. J. Experiments in Fluids, p 1-9 (2011).

[5] Harris D.M., Miller V.A., and Williamson C.H.K.: A short wave instability caused by the approach of a vortex pair to a ground plane. J. Physics of Fluids, v 22, n 9, Sep-tember 30 (2010). 